\title{
Ovarian Drilling Efficacy, Estradiol Levels and Pregnancy Rate in Females With Polycystic Ovary Syndrome
}

\author{
Farideh Moramezi ${ }^{1, *} ;$ Roshan Nikbakhat $^{1}$; Azam Rafigh ${ }^{1}$ \\ ${ }^{1}$ Fertility and Perinatology Research Center, Imam Khomeini Hospital, School of Medicine, Ahvaz Jundishapur University of Medical Sciences, Ahvaz, IR Iran \\ ${ }^{*}$ Corresponding author: Farideh Moramezi, Fertility and Perinatology Research Center, Imam Khomeini Hospital, School of Medicine, Ahvaz Jundishapur University of Medical Sci- \\ ences, Ahvaz, IR Iran. Tel: +98-9161115368, Fax: +98-6113335399, E-mail: farideh330@yahoo.com
}

Received: June 17, 2014; Revised: October 15, 2014; Accepted: December 28, 2014

\begin{abstract}
Background: Polycystic ovary syndrome (PCOS) is the most common cause of oligoovulation and anovulation in general population and in females with infertility.

Objectives: The purpose of this study was to compare the efficacy of ovarian laparoscopic drilling procedure (LOD) in females with PCOS, resistant to treatment with estradiol (E2) level less than $40 \mathrm{pg} / \mathrm{mL}$ versus more than $40 \mathrm{pg} / \mathrm{mL}$.

Materials and Methods: Females with PCOS, resistant to drug for ovary stimulation, were grouped based on the Estradiol levels of $\leq 40$ $\mathrm{pg} / \mathrm{mL}(\mathrm{n}=13)$ and $>40 \mathrm{pg} / \mathrm{mL}(\mathrm{n}=15)$. To survey the ovulation, continuing spontaneous ovulation and cumulative pregnancy rate, ovarian laparoscopic drilling was carried out after the analysis of serum E2.

Results: There was significant difference in the average starting time of ovulation and continuing spontaneous ovulation of cases with PCOS with E2 levels $>40 \mathrm{pg} / \mathrm{mL}$, compared with ones with $\mathrm{E} 2 \leq 40 \mathrm{pg} / \mathrm{mL}(\mathrm{P}=0.029, \mathrm{P}=0.05$, respectively). Significant differences were also found in pregnancy rates of cases with PCOS with E2 levels $>40 \mathrm{pg} / \mathrm{mL}$ compared with ones with E2 $\leq 40 \mathrm{pg} / \mathrm{mL}(\mathrm{P}=0.05)$.

Conclusions: This study revealed that LOD in females with PCOS with a serum E2 $>40 \mathrm{pg} / \mathrm{mL}$ was sufficient and safe to trigger development of ovarian follicles followed by clinical pregnancy.
\end{abstract}

Keywords:Estradiol; Treatment; Pregnancy

\section{Background}

Polycystic ovary syndrome (PCOS) is the most common cause of oligoovulation and anovulation in the general population and in females with infertility (1). The diagnosis of PCOS can be confirmed by ruling out other medical disorders and it is based on two of the following disorders; 1- Oligoovulation or anovulation, manifested as oligomenorrhea or amenorrhea, 2- Hyperandrogenism, 3- Hyperandrogenism and its clinical complaints, 4- polycystic ovaries diagnosed by ultrasound (2-4). In other words, polycystic ovaries is a result of a functional disorder and does not occur due to central or local defects (4). In patients with persistent anovulation, the average daily level of estrogen and androgen is high which depends on the persistent stimulatory effect on secretion of the luteinizing hormone (LH) (5). This, in turn, has caused an increase in the average daily levels of testosterone, androstenedione, dehydroepiandrosterone (DHEA), dehydroepiandrosterone sulfate (DHEAS), 17- hydroxyprogesterone (17-OHP) and estrone (6). In addition, in cases with persistent anovulation, the ovaries do not secrete more estrogen and the estradiol concentration is permanently equivalent with its concentration in the early follicular phase (7). Indeed, despite a reduced secretion of estradiol, free estradiol levels increase, due to a significant decrease in sex hormone-binding globulin (SHBG) secretion (7). Properly, an elevated LH-to-follicle-stimulating hormone (FSH) ratio is associated with the increase of free estradiol secretion (8). Appropriate selection of patients for treatment is an important factor in successful induction of ovulation in patients with PCOS. At first, PCOS treatments include weight loss, clomiphene citrate, gonadotropins administration and surgical treatment. Indeed, there is no fixed protocol for induction of ovulation in cases with anovulation (8). However, the rational method that must be used is first the least invasive and then the most aggressive method. Therefore, routinely, in patients with normal thyroid function, normal prolactin levels, no galactorrhea and with mean serum estradiol levels of more than $40 \mathrm{pg} /$ $\mathrm{mL}$ or natural response of menstruation to progesterone concentration, clomiphene is the drug of choice for ovulation induction (9). However, for ovulation induction in cases with PCOS who are resistant to medical procedures, the surgical methods can be performed (10). The primary surgical methods for ovulation induction management of PCOS was ovarian wedge resection, taken place to reduce the size of the androgen-producing tissue, and thus, to decrease the disturbances of hyperandrogenism (10). In $91 \%$ of patients with PCOS treated by this method, it was as-

Copyright (C) 2015, Ahvaz Jundishapur University of Medical Sciences. This is an open-access article distributed under the terms of the Creative Commons Attribution-NonCommercial 4.0 International License (http://creativecommons.org/licenses/by-nc/4.0/) which permits copy and redistribute the material just in noncommercial usages, provided the original work is properly cited. 
sociated with the resumption of menstruation. However, pelvic adhesion is the side-effect of this procedure which may lead to iatrogenic infertility, i.e. by uterine/tubal factors (11). As an alternative, laparoscopy techniques compared with this procedure are less associated with the formation of adhesions in the pelvis. Moreover, it was observed that in laparoscopic ovarian diathermy (LOD) (or puncture in the ovary), the ovulation rate increased and the resistant to induction by standard ovulating agents decreased (12). Additionally, surgical treatment resulted in a significant reduction in serum levels of $\mathrm{LH}$, testosterone, DHEAS and androstenedione and the ratio of LH to FSH. In treatment of PCOS, different techniques for laparoscopic ovarian tissue destruction have been described, i.e. laser, monopolar and bipolar cautery (12). LOD appears to be an effective minimally invasive procedure and useful for the induction of ovulation in patients with PCOS, resistant to clomiphene citrate, seeking pregnancy (13). Moreover, LOD has been effective on assisted reproductive techniques (ART) outcomes (13). Although randomized trials have shown that no technique is superior to other methods, A number of recent reports have supported the effectiveness of diathermy or electrocautery to treat patients with PCOS resistant to medical methods, i.e. clomiphene citrate or gonadotropins $(13,14)$, given that ovulation in patients with PCOS with estradiol levels greater than $40 \mathrm{pg} / \mathrm{mL}$ occurs better.

\section{Objectives}

The purpose of this study was to investigate the effects of treatment with LOD on reproductivity in females with PCOS, resistant to treatment with estradiol levels of $\leq$ or $>40 \mathrm{pg} / \mathrm{mL}$.

\section{Materials and Methods}

\subsection{Study Design}

A comparative, randomized, clinical trial was carried out in Fertility and Infertility Center of Imam Khomeini Teaching Hospitals in Ahvaz, Iran. This study was performed between April 2011 and January 2013 and targeted the infertility patients referred to the hospital with complaints of infertility, polycystic ovarian syndrome, resistance to ovulation induction, and candidate for laparoscopic ovarian drilling procedure. The Ethics Committee of Jundishapur Ahvaz University of Medical Sciences approved this study. Patients eligible to participation in this study during the 18 months in the hospital for elective infertility cauterization surgery were selected. The serum estradiol level was measured. Fifteen patients had serum estradiol levels more than $40 \mathrm{pg} / \mathrm{mL}$ and 13 had lower levels. Afterwards, the subjects underwent laparoscopic procedures for ovarian cauterization. Serial transvaginal ultrasounds were performed every month for monitoring the ovulation and pregnancy rate. The number of postoperative patients in which spontaneous ovulation and clinical pregnancy oc- curred was also analyzed. The inclusion criteria were: irregular menstruation, clinical and biochemical evidences of hyperandrogenemia, sonographic evidence of PCOS, i.e. high rate of microcysts (0.5 - 0.8 inch) and resistance to treatment (failure of ovulation induction with clomiphene-letrozole-metformin-gonadotropins-tamoxifen after 6-3 months). Patients with the following criteria were not enrolled in the present study: $\mathrm{FSH} \geq 12$, family history of premature ovarian failure, smoking, and age over 40 years. The exclusion criteria also included medical conditions that precluded surgery or anesthesia, lack of patient cooperation, pregnancy, and dissatisfaction of patients from the operation or enrollment in the study.

\subsection{Laparoscopic Ovarian Drilling Technique}

Laparoscopic surgery for ovarian drilling (LOD) was performed under general anesthesia. One $10-\mathrm{mm}$ port and two 5-mm ports were taken in the infraumbilical region and on either side of the lower abdomen, respectively. Four holes within four seconds, with a depth of 4-3 mm were created in both ovaries. By observation of the pelvic cavity, all other causes of infertility were investigated. During the laparoscopy, washing and suctioning was performed with $500 \mathrm{~mL}$ of Ringer's to prevent postoperative adhesions. Patients who underwent LOD were then treated with ovulation induction drugs after a following period of four months to control the spontaneous ovulation and clinical pregnancy. The first visit of the patients was carried out in the first cycle after cauterization, in which occurrence or non-occurrence of ovulation was confirmed by ultrasound and up to four months in each cycle, patients underwent ultrasound test.

\subsection{End Points}

The end point of the study was to see whether triggering the treatment with laparoscopic cauterization procedure had any effect on reproductive outcome of the patients with PCOS, resistant to drug treatment.

\subsection{Statistics Analysis}

All the analyses were carried out with the SPSS 16 statistical software. For comparison of the rates of ovulation and clinical pregnancy, chi-squared test was used. Categorical variables were used to compare the average times of ovulation and clinical pregnancy between the groups, using Independent t test, ${ }^{2}$ tests or Fisher's exact test, where appropriate. For all other outcomes, a nominal P value < 0.05 was considered significant.

\section{Results}

\subsection{Maternal Demographic Characteristics Assess- ment}

A total of 28 cases were randomized and available for investigation. The demographic characteristics are shown 
in Table 1. None of the women refused to participate in the study. The mean age of all of the patients was $25.5 \pm$ 4.4 years with a range of 16 to 36 years. There was no significant relationship regarding the mean ages between the group with E2 $<40 \mathrm{pg} / \mathrm{mL}$ versus E2 $>40 \mathrm{pg} / \mathrm{mL}(\mathrm{P}=$ $0.85)$. Of 13 women with E2 < 40 pg/mL, 10 (76.9\%) were nulliparous and 3 (23.1\%) were multiparous, whereas from 15 women with E2 > $40 \mathrm{pg} / \mathrm{mL}$, 11 (73.3\%) were nulliparous and 4 (26.7\%) were multiparous. However, the relation between parity and the amount of E2 was not significant $(\mathrm{P}=0.99)$. The two groups were identical in body mass index (BMI) and menstrual pattern (oligomenorrhea and regular menstrual cycle). We recorded no complications or difficulties in the performance of laparoscopy (none of the females had abdominal pain or nausea). In case of drug treatment before undergoing laparoscopy, from 13 patients with E2 < $40 \mathrm{pg} / \mathrm{mL}, 12$ (92.2\%) were given clomiphene, 7 (53.8\%) human menopausal gonadotropin (HMG), 13 (53.8\%) tamoxifen, 1 (53.8\%) letrozole, 9 (53.8\%) metformin, 1 (53.8\%) gonal $\mathrm{F}$, and 1(53.8\%) menogan. From 15 patients with E2 > $40 \mathrm{pg} /$ mL, 12 (92.2\%) were given clomiphene, 7 (53.8\%) HMG, 13 (53.8\%) tamoxifen, 1 (53.8\%) letrozole, 9 (53.8\%) metformin, 1 (53.8\%) gonal F, and 1 (53.8\%) menogan. Seventy percent of the cases with PCOS withE2 $<40 \mathrm{pg} / \mathrm{mL}$ had primary infertility and the remaining had secondary infertility; eleven withE2 $>40 \mathrm{pg} / \mathrm{mL}$ had primary infertility and the remaining had secondary infertility. The mean duration of infertility among the participants was at least two years, so that in the patients with $\mathrm{E} 2<40 \mathrm{pg} / \mathrm{mL}$ it was $7.8 \pm 4.4$ and in cases with E2 $>40 \mathrm{pg} / \mathrm{mL}$ it was $6.2 \pm 4.4$. Generally, The most common cause of infertility in both groups (E2 $>40$ and E2 > 40) was ovarian failure (100\%); other causes along with ovarian failure were tubal failure $(7.7 \%$ and $6.7 \%$, respectively), male factor ( $46.2 \%$ and $40 \%$, respectively), and uterine defects (15\% and 6.4, respectively). The most common cause of infertility in the $\mathrm{E} 2<40 \mathrm{pg} / \mathrm{mL}$ group was ovarian failure (100\%); other causes along with ovarian failure were tubal failure (16.7\%), uterine defects, and uterine defects along with ovarian failure (15\%). All the causes that were along with ovarian failure had been treated before performing the laparoscopic cauterization treatment.

\subsection{Clinical Parameters Assessment}

The clinical parameters characteristics are shown in Tables 2 and 3. The average starting time of ovulation in patients after laparoscopic cauterization treatment in the E2 $<40 \mathrm{pg} / \mathrm{mL}$ group was $2.4 \pm 2$ months and in the other group was $1.3 \pm 0.6$ months. Therefore, the relationship between the average starting time of ovulation and the amount of serum E2 was significant. Indeed, the average starting time of ovulation in patients with $\mathrm{E} 2<40 \mathrm{pg} / \mathrm{mL}$ was longer than the ones with $\mathrm{E} 2>40 \mathrm{pg} / \mathrm{mL}(\mathrm{P}=0.0002)$. The mean percentage in the group with E2 $>40 \mathrm{pg} / \mathrm{mL}$ was $100 \%$, but in cases with E2 $<40 \mathrm{pg} / \mathrm{mL}$ it was $84.6 \%$. The cumulative pregnancy rate (60\%) in the group with E2 $<40$ $\mathrm{pg} / \mathrm{mL}$ was more pronounced than the group with E2 $>40$ $\mathrm{pg} / \mathrm{mL}(15.4 \%)$. The mean duration of occurrence of clinical pregnancy after the laparoscopic cauterization treatment in cases with $\mathrm{E} 2<40 \mathrm{pg} / \mathrm{mL}$ was $2 \pm 45 / 2$ months and in the group with $\mathrm{E} 2>40 \mathrm{pg} / \mathrm{mL}$ it was $1.3 \pm 0.6$ months.

Table 1. Maternal Demographic and Clinical Characteristics in Females Admitted to the Department ${ }^{\text {a }}$

\begin{tabular}{|c|c|c|c|}
\hline & \multicolumn{2}{|c|}{ Groups } & \multirow[t]{2}{*}{ P Value } \\
\hline & Estradiol $<40 \mathrm{pg} / \mathrm{mL}$ & Estradiol $>40 \mathrm{pg} / \mathrm{mL}$ & \\
\hline Cases, No. & 15 & 13 & 0.865 \\
\hline Age, $y$ & $26.9 \pm 4.2$ & $27.2 \pm 3.1$ & 0.85 \\
\hline BMI, $\mathrm{kg} / \mathrm{m}^{2}$ & $29.5 \pm 3.1$ & $30.4 \pm 3.4$ & 0.486 \\
\hline Primary sterility & $10(76.9)$ & $11(73.3)$ & 0.89 \\
\hline Secondary sterility & $3(23.1)$ & $4(26.7)$ & 0.437 \\
\hline Duration of infertility & $6.2 \pm 4.4$ & $7.8 \pm 4.2$ & 0.050 \\
\hline Clomiphene treatment & 10 & 15 & 0.758 \\
\hline HMG treatment & 7 & 9 & 0.396 \\
\hline Tamoxifen treatment & 13 & 15 & 0.879 \\
\hline Letrozole treatment & 1 & 15 & 0.0001 \\
\hline Metformin treatment & 9 & 10 & 0.798 \\
\hline Gonal F treatment & 1 & 4 & 0.09 \\
\hline Menogan treatment & 1 & 2 & 0.978 \\
\hline Male factor of infertility & 6 & 6 & - \\
\hline Ovarian factor of infertility & 13 & 15 & 0.750 \\
\hline Oviduct tubal factor of infertility & 1 & 1 & - \\
\hline Uterus factor of infertility & 2 & 1 & 0.958 \\
\hline Idiopathic factor of infertility & 0 & 0 & - \\
\hline Duration of Ovulation, mon & $2.54 \pm 1$ & $1.33 \pm 0.6$ & 0.029 \\
\hline Duration of Pregnancy, mon & $6.19 \pm 1$ & $3.25 \pm 0.6$ & 0.05 \\
\hline Ovulation rate, number & 11 & 15 & 0.579 \\
\hline
\end{tabular}

a Abbreviations: BMI, body mass index; HMG, human menopausal gonadotropin. 
Moramezi F et al.

\begin{tabular}{lccc}
\hline Table 2. Comparison of Serum Estradiol, Insulin, FSH, FBS, HDL, LH and GTT Between non- and Treated Cases & \\
\hline Duration of Ovulation & $\mathbf{E 2}<\mathbf{4 0} \mathbf{~ p g} / \mathbf{m L}$ & $\mathbf{E 2}>\mathbf{4 0} \mathbf{~ p g} / \mathbf{m L}$ & P Value \\
\hline $\mathbf{1}$ month & 3 & 11 & 0.002 \\
$\mathbf{2}$ months & 2 & 3 & 0.765 \\
3 months & 6 & 1 & 0.001 \\
$\mathbf{4}$ months and more & 0 & 0 & - \\
Total & 11 & 15 & 0.624 \\
\hline
\end{tabular}

Table 3. Comparison of Serum Estradiol, Insulin, FSH, FBS, HDL, LH and GTT Between non- and Treated Cases

\begin{tabular}{lccc}
\hline Duration of Ovulation & E2 $<\mathbf{4 0} \mathbf{~ p g} / \mathbf{m L}$ & E2 $>\mathbf{4 0} \mathbf{~ p g} / \mathbf{m L}$ & P Value \\
\hline 1 month & 0 & 1 & 0.89 \\
$\mathbf{2}$ months & 0 & 1 & 0.89 \\
3 months & 0 & 1 & 0.89 \\
$\mathbf{4}$ months and more & 2 & 6 & 0.024 \\
Total & 2 & 9 & 0.001 \\
\hline
\end{tabular}

\section{Discussion}

PCOS is the most common cause of oligoovulation and anovulation in the general population and in infertile females. Properly, infertile females with anovulation are candidates for ovulation induction $(2,3)$. First choice of treatment for this problem, especially in patients with more $40 \mathrm{pg} / \mathrm{mL}$ serum E2 levels is using ovarian-stimulation drugs (4). However, clinicians are often faced with the problem of how to manage the induction of ovulation with a variety of drugs, which may lack specificity, low efficiency, or have potentially severe side-effects for the subjects or the ovarian function. For instance, one of the most commonly used drugs to stimulate the development of ovarian follicles is clomiphene citrate (15). Nevertheless, some patients do not respond to an adequate trial of this drug $(13,14)$. Therefore, proper steps should be taken to choose a new avenue, based on using the laparoscopy procedure, which can help the subjects with PCOS to overcome infertility. The results of the present study showed that after treatment with LOD, the females with anovulation resistant to drug treatment, especially the ones with E2 $>40 \mathrm{pg} / \mathrm{mL}$, were better capable of inducing follicular maturation and ovulation. Indeed, the starting time of ovulation after laparoscopy in patients with PCOS with E2 $\leq 40 \mathrm{pg} / \mathrm{mL}$ was longer than the ones with E2 $>40 \mathrm{pg} / \mathrm{mL}$. Therefore, it seems that there is a meaningful relationship between the average starting time of ovulation and the amount of serum E2. In addition, the mean rate of ovulation in the group with E2 > $40 \mathrm{pg} / \mathrm{mL}$ was more pronounced than the group with E2 $<40 \mathrm{pg} / \mathrm{mL}$. However, whether the quality of the follicles released in these forms is optimal as well as the incidence of premature ovarian failure stays to be clarified. These findings were also in accordance with other three studies by Gjonnaess (16), Amer et al. (17) and Al-Ojaimi (18). Gjonnaess (16) showed that LOD was effective and well tolerated for induction of ovulation in patients with PCOS undergoing assisted reproductive techniques (ART) treatment. Amer et al. (17) have also found similar clinical outcomes with LOD during ART treatment in abnormal responder patients. Balen et al. (9)obtained the same successes in inducing follicular maturation and ovulation rates in females treated with LOD before undergoing ART treatment. In contrast, Abdel Gadir et al. (19) reported that LOD was not more efficient to achieve optimal oocyte maturation than other treatment procedures. These observations strongly indicate that the effect of surgery is mediated via a central mechanism rather than a direct effect on the treated ovary (20). However, the pathophysiology of unusual ovarian blood supply in PCOS is not evidently understood. One probable reason is that E2 may play a role in the moderation of vascularity of ovary and uterine (21). Greenblatt and Casper (5) reported that the level of E2 at the first day after the LOD dropped, so that it reached the minimum concentration by day four and thereafter began to rise steadily to the end of the first menstruation period. Therefore, the suggestion of any relationship between the serum E2 levels and ovarian blood supply alters stays elusive. Alternatively, a noteworthy decline in vascular impedance to blood supply in the ovarian artery (22) and in the vessels around the follicles, in correlation with an increase in the number of follicles and serum E2 concentration (23), was observed after the ovarian stimulation with gonadotropins. Considering these observations and the data reported by Zaidi (24), it seems that some factors other than E2 could be the cause of increased ovarian stromal vascularity in PCOS. Primordial or smaller preantral follicles do not have any special vascular supply of their own and derive their blood supply from the stromal blood vessels (5). Subsequent growth of primary follicles leads to development of a vascular 
network with increased follicular blood flow. Hence, the stromal blood flow velocity in an inactive or quiescent ovary may reflect the baseline blood flow perfusion. LOD may result in a reduction in the number of small and intermediate follicles usually seen in PCOS; it has the same effect on the ovarian stromal tissue $(25,26)$. Regarding these effects as well as the abovementioned mechanism by which ovarian stromal blood flow declined after the gonadotropin-releasing hormone agonist (GnRH) therapy (5), we can hypothesize that the decline in ovarian stromal blood flow velocity could be the result of the direct electrical and/or thermal effects of LOD. Taking into consideration the rise of ovarian stromal blood flow velocity in PCOS $(27,28)$ and its possible effects on ovarian steroidogenesis, there might be a possible beneficial effect of diminished ovarian stromal blood flow velocity on ovarian steroidogenesis in PCOS. In the present experiment, the difference was shown in terms of the cumulative ongoing pregnancy rate in patients with PCOS with E2 levels higher or lower than $40 \mathrm{pg} / \mathrm{mL}$, undergoing LOD treatment. In patients with PCOS with E2 $>40 \mathrm{pg} / \mathrm{mL}$, the pregnancy rate increased four times. Furthermore, other studies have been able to find the positive influence of LOD treatment on reproductive outcomes $(28,29)$. In contrast, other studies have reported that reproductive parameters such as clinical pregnancy, were not significant in patients with E2 levels both higher and lower than 40 $\mathrm{pg} / \mathrm{mL}(30,31)$. Considering this prospective randomized experiment, we can conclude that LOD, especially in females with PCOS with a serum E2 higher than $40 \mathrm{pg} / \mathrm{mL}$ is sufficient and safe to trigger ovulation followed by clinical pregnancy. However, well-designed, further studies are essential to say a final conclusion.

\section{Acknowledgements}

The present study was extracted from the medical doctoral thesis of Dr. Azam Rafigh. The authors wish to acknowledge the efforts of Dr. Masoud Hemadi (vice chief of Fertility, Infertility and Perinatology Research Center, Ahvaz Jundishapur University of Medical Sciences (AJUMS), Ahvaz, Iran) for his generous help in processing the study.

\section{Funding/Support}

This study was supported by a research grant from Ahvaz Jundishapur University of Medical Sciences, Ahvaz, Iran (Grant No. D/520).

\section{References}

1. Anwary SA, Alfazzaman M, Begum NA. Clinical study on PCOS patients in a terthiary hospital. Med Today. 2009;22(1):34-6.

2. Abdellah MS. Reproductive outcome after letrozole versus laparoscopic ovarian drilling for clomiphene-resistant polycystic ovary syndrome. Int J Gynaecol Obstet. 2011;113(3):218-21.

3. Shokeir T, El-Kannishy G. The effects of laparoscopic ovarian drilling on flow mediated dilation in women with PCOS: relationship with insulin resistance and IGF-I. Minerva Ginecol. 2014; 66(2):209-18.
4. Felemban A, Tan SL, Tulandi T. Laparoscopic treatment of polycystic ovaries with insulated needle cautery: a reappraisal. Fertil Steril. 2000;73(2):266-9.

5. Greenblatt E, Casper RF. Endocrine changes after laparoscopic ovarian cautery in polycystic ovarian syndrome. Am J Obstet Gynecol. 1987;156(2):279-85.

6. Lemieux S, Lewis GF, Ben-Chetrit A, Steiner G, Greenblatt EM. Correction of hyperandrogenemia by laparoscopic ovarian cautery in women with polycystic ovarian syndrome is not accompanied by improved insulin sensitivity or lipid-lipoprotein levels. J Clin Endocrinol Metab. 1999;84(11):4278-82.

7. Vause TD, Cheung AP, Sierra S, Claman P, Graham J, Guillemin JA, et al. Ovulation induction in polycystic ovary syndrome. J Obstet Gynaecol Can. 2010;32(5):495-502.

8. Rossmanith WG, Keckstein J, Spatzier K, Lauritzen C. The impact of ovarian laser surgery on the gonadotrophin secretion in women with polycystic ovarian disease. Clin Endocrinol (Oxf). 1991;34(3):223-30.

9. Balen AH, Jacobs HS. A prospective study comparing unilateral and bilateral laparoscopic ovarian diathermy in women with the polycystic ovary syndrome. Fertil Steril. 1994;62(5):921-5.

10. Alborzi S, Khodaee R, Parsanejad ME. Ovarian size and response to laparoscopic ovarian electro-cauterization in polycystic ovarian disease. Int J Gynaecol Obstet. 2001;74(3):269-74.

11. Lazovic G, Milacic D, Terzic M, Spremovic S, Mitijasevic S. Medicaments or surgical therapy of PCOS. Fertil Steril. 1978;70:472.

12. Vegetti W, Ragni G, Baroni E, Testa G, Marsico S, Riccaboni A, et al., editors. Laparoscopic ovarian drilling versus low-dose pure FSH in anovulatory clomiphene-resistant patients with polycystic ovarian syndrome: randomized prospective study.; Human Reproduction.; 1998; England. Oxford Univ Press; p. 120.

13. Farquhar CM, Williamson K, Gudex G, Johnson NP, Garland J, Sadler L. A randomized controlled trial of laparoscopic ovarian diathermy versus gonadotropin therapy for women with clomiphene citrate-resistant polycystic ovary syndrome. Fertil Steril. 2002;78(2):404-11.

14. Daya S. Laparoscopic ovarian electrocautery in the treatment of clomiphene-resistant polycystic ovary syndrome. Evid Based $\mathrm{Ob}$ stet Gynecol. 2001;3(3):164-5.

15. Cohen J. Laparoscopic procedures for treatment of infertility related to polycystic ovarian syndrome. Hum Reprod Update. 1996;2(4):337-44.

16. Gjönnaess H. Polycystic ovarian syndrome treated by ovarian electrocautery through the laparoscope. Fertil Steril. 1984;41(1):20-5.

17. Amer SA, Li TC, Ledger WL. Ovulation induction using laparoscopic ovarian drilling in women with polycystic ovarian syndrome: predictors of success. Hum Reprod. 2004;19(8):1719-24.

18. Al-Ojaimi EH. Pregnancy outcomes after laparoscopic ovarian drilling in women with polycystic ovarian syndrome. Saudi Med J. 2006;27(4):519-25.

19. Abdel Gadir A, Mowafi RS, Alnaser HM, Alrashid AH, Alonezi OM, Shaw RW. Ovarian electrocautery versus human menopausal gonadotrophins and pure follicle stimulating hormone therapy in the treatment of patients with polycystic ovarian disease. Clin Endocrinol (Oxf). 1990;33(5):585-92.

20. Armar NA, Lachelin GC. Laparoscopic ovarian diathermy: an effective treatment for anti-oestrogen resistant anovulatory infertility in women with the polycystic ovary syndrome. Br J Obstet Gynaecol.1993;100(2):161-4.

21. Arefi X. LOD in metformin and c.c resistant PCOS.JFamily Reprod Health.;2(1):17-21.

22. Gomel V, Yarali H. Surgical treatment of polycystic ovary syndrome associated with infertility. Reprod Biomed Online. 2004;9(1):35-42.

23. Butt F. Laparoscopic ovarian drilling by diathermy for ovulation induction in infertile women with polycystic ovarian syndrome. Ann King Edward Med Univ. 2011;17(2):150-6.

24. Zaidi J. Blood flow changes in the ovarian and uterine arteries in women with normal and polycystic ovaries. Hum Fertil (Camb). 2000;3(3):194-8.

25. Deutinger J, Reinthaller A, Bernaschek G. Transvaginal pulsed 


\section{Moramezi F et al.}

Doppler measurement of blood flow velocity in the ovarian arteries during cycle stimulation and after follicle puncture. Fertil Steril. 1989;51(3):466-70.

26. Dolz M, Osborne NG, Blanes J, Raga F, Abad-Velasco L, Villalobos A, et al. Polycystic ovarian syndrome: assessment with color Doppler angiography and three-dimensional ultrasonography. J Ultrasound Med.1999;18(4):303-13.

27. Findlay JK. Angiogenesis in reproductive tissues. $J$ Endocrinol. 1986;111(3):357-66.

28. Liguori G, Tolino A, Moccia G, Scognamiglio G, Nappi C. Laparoscopic ovarian treatment in infertile patients with polycystic ovarian syndrome (PCOS): endocrine changes and clinical out- come. Gynecol Endocrinol. 1996;10(4):257-64.

29. Battaglia C, Artini PG, D'Ambrogio G, Genazzani AD, Genazzani AR. The role of color Doppler imaging in the diagnosis of polycystic ovary syndrome. Am J Obstet Gynecol. 1995;172(1 Pt 1):108-13.

30. Parsanezhad ME, Bagheri MH, Alborzi S, Schmidt EH. Ovarian stromal blood flow changes after laparoscopic ovarian cauterization in women with polycystic ovary syndrome. Hum Reprod. 2003;18(7):1432-7.

31. Safdarian L, Eslamian L, Adineh M, Aghahoseini M, Aleyasin A, Saidi H. Impact of laparascopic ovarian electrocautery on doppler indices women stromal blood flow in women with polycystic ovary syndrome. Acta Med Iran. 2008;46(3):51-9. 